\title{
GRUPOS ÉTNICOS Y SISTEMA GENERAL DE REGALÍAS EN EL CONTEXTO DE LOS DERECHOS HUMANOS EN COLOMBIA
}

The ethnic groups and general system of royal ties in the

context of human rights in Colombia

Elba Del Carmen Donado Arrieta ${ }^{1}$

\section{RESUMEN}

Ante el problema social reflejado en gran parte de las minorías étnicas de Colombia, debido principalmente al desplazamiento forzoso y conociendo la problemática de su hábitat, derivada de la explotación de los recursos naturales no renovables, el objetivo de este artículo es analizar a los grupos étnicos de Colombia en relación con el Sistema General de Regalías (SGR) en el contexto de los derechos humanos. Este trabajo se perfila por una metodología de carácter cualitativo y se apoya en la técnica del análisis textual de documentos. Se puede concluir que las reformas del SGR se orientan hacia la equidad social y económica de todos los lugares geográficos involucrados y la forma de retribuir las ganancias, incluyendo a las diferentes etnias. No obstante, los grupos étnicos han sido vulnerados en sus derechos fundamentales.

Palabras clave: Desplazamiento forzoso, hábitat, minoría étnica, recursos naturales no renovables, regalías.

\begin{abstract}
Assuming the social problem reflected in most of ethnic minorities groups in Colombia, mainly due to the forced displacement and approaching to the several difficulties that they as a community have in their own habitat and affected from the exploitation of non-renewable natural resources. The main objective of this research is to analyze the ethnic groups of Colombia in relation to the General System of Royalties (GSR) in the context of human rights. This work uses a qualitative methodology and is based on the technique of textual analysis of documents. The development of this research
\end{abstract}

\footnotetext{
${ }^{1}$ Abogada de la Institución Universitaria de Envigado, Administrador del Comercio Exterior de la Universidad de Bogotá Jorge Tadeo Lozano - seccional del caribe, especialista derecho procesal penal de la Universidad Autónoma Latinoamericana -UNAULA-, Doctor en Derecho (c) de la Universidad de Baja California. E-mail: elbadonadoarrieta@gmail.com
} 
can conclude that the different changes of the GSR are focus to the social and economic equality for all the part involved on the system and the way that revenues are distribute. However, the ethnic groups fundamental rights are still being transgress.

Keywords: Forced displacement, habitat, ethnic minority, non-renewable natural resources, Royalties.

\section{INTRODUCCIÓN}

Los grupos étnicos en Colombia suman aproximadamente unas 87 etnias o pueblos que preservan sesenta y cuatro lenguas autóctonas y cerca de trescientas formas dialécticas, situados en diversas regiones del país, como el Archipiélago de San Andrés, Providencia y Santa Catalina, con raíces culturales afro-anglo-antillanas; el municipio de San Basilio de Palenque Departamento de Bolívar, donde se habla el palanquero y lenguaje criollo; entre otras regiones. Los departamentos con mayor porcentaje de indígenas son Guainía, Vaupés, La Guajira, Amazonas, Vichada, Cauca y Nariño. En La Guajira, Cauca y Nariño concentran aproximadamente la mitad de los indígenas del país. El Departamento Nacional de Estadística (DANE, 2007:37) dio a conocer que en Colombia residen 1.392.623 indígenas representando el 3,43\% de la población. Por su parte, los afrocolombianos alcanzan las 4.311 .757 personas, constituyendo el 10,62\% de la población colombiana. Aparte se suma el pueblo Rom o gitano con 4.858 personas, siendo el 0,01\% en el país. En síntesis, todo el grupo étnico de Colombia corresponde al 14,06\% de la población.

Sin embargo, en un censo reciente, el DANE (2018) devela el crecimiento significativo de la población indígena del país. De esta forma, haciendo uso de la metodología de auto reconocimiento, para determinar la pertenencia étnica, con el Censo Nacional Colombiano de Población y Vivienda del año 2018, se han identificado 115 pueblos indígenas nativos, a diferencia de las cantidades determinadas en los censos anteriores. El incremento actual es de 22 pueblos correspondientes a nuevos grupos étnicos o pueblos indígenas en zonas fronterizas

De la misma manera, este Censo aporta información valiosa sobre el reconocimiento de los cuatro pueblos indígenas más numerosos del país, como lo son, las etnias Wayuu, Zenú, Nasa y Pastos, quienes concentran el 58,1\% de la población indígena del País. (DANE-DCD, CNPV, 2018). Los indígenas pasaron de representar el 3,4\% de la población colombiana, al 4,4\% de la misma, teniendo su mayor representatividad en los Departamentos de La Guajira, Cauca, Nariño y Córdoba. Así se observa que los grupos étnicos de Colombia pasaron de ser 93 en años anteriores, para ser actualmente unos 115, de acuerdo al último censo de la nación (Dane, 2018).

Según el DANE (2007:23), los indígenas han sufrido un proceso migratorio hacia las ciudades y los pueblos cabecera de municipio en razón a los cambios culturales, el desplazamiento forzoso por parte de actores del conflicto armado colombiano, debido a que han sido víctimas de asesinatos, secuestros a raíz de la lucha de la tierra correspondiente a los resguardos, especialmente en las 
regiones de la Sierra Nevada de Santa Marta, del Urabá y en los departamentos de Cauca, Córdoba, Guaviare, Nariño y Putumayo (DANE,2007:20).

Gran parte de las actividades económicas desarrolladas en las inmediaciones del entorno de estos grupos minoritarios, han tenido una trascendencia drástica que ha demeritado la calidad de vida de las etnias, cuando las explotaciones de metales como el oro, plata y platino, entre otras actividades industriales, han ido mermando su medio ambiente. De acuerdo con los planteamientos de Acosta, (2017:79), la humanidad se está concientizando sobre el uso que hace del medio ambiente y, por ello, se están sumando esfuerzos que propendan por la protección de los recursos naturales, con la intención de poder formular políticas gubernamentales cada vez más favorables.

A partir de ello, hoy en día se tiene en cuenta la calidad no solo de los recursos naturales renovables sino también los no renovables, revisando y regulando las actividades industriales con la finalidad de preservar las condiciones óptimas del medio ambiente, la atmósfera, el aire que respiramos, los ríos, los animales, vegetales, plantas en general y a todos los seres vivos que habitan la tierra. En este sentido, gran parte de los derechos humanos- DDHH se refieren a las condiciones de vida digna para estos grupos étnicos, cabe destacar la vigilancia y procura de su hábitat en condiciones adecuadas, es un punto permanente de consideración.

Por otra parte, uno de los problemas latentes en las regiones indígenas es el desplazamiento forzoso, a consecuencia de los conflictos derivados por los grupos armados al margen de la ley, las actividades comerciales ilegales y el narcotráfico, entre otros aspectos. Desde el ámbito de los DDHH, se destaca que los grupos étnicos de Colombia han estado sufriendo diferentes afrentas contra su calidad de vida, incluyendo más allá de los desplazamientos forzados, el deterioro sistemático de su hábitat.

Al respecto, la Constitución Política de Colombia de 1991 refrenda el derecho a una vida digna en su artículo 11 (Salgado, 2017). Además, el derecho de conservación del medio ambiente está regulado por los artículos 79 y 89 de la mencionada carta magna y Decreto 2811 de 1974, por el cual se dicta el Código Nacional de Recursos Naturales Renovables y de Protección al Medio Ambiente; y, cuando se atenta contra las condiciones del medio ambiente, en este caso se está afectando tanto a los grupos étnicos, como al resto de la población colombiana. Uno de los aspectos legales normativos de Colombia, encaminado a la retribución que deben hacer las empresas al Estado cuando desarrollan actividades de explotación de recurso naturales no renovables, tiene que ver con el Sistema General de Regalías SGR. Este sistema, entre sus regulaciones, considera para los habitantes colombianos la forma en que tales regalías pueden contribuir al beneficio de su calidad de vida.

Es por ello que el propósito de este artículo es describir la problemática de los grupos étnicos y el Sistema General de Regalías en el contexto de los Derechos Humanos en Colombia. Este documento está estructurado por una introducción, un cuerpo teórico que presenta antecedentes y bases teórico 
científicas, además la parte legal normativa de SGR en Colombia y las reflexiones finales.

\section{Referentes teóricos}

Los testimonios indios transitaron desde el inicio de la conquista y de la época de la colonia entre 1550 y 1810 a la llamada etapa de la Independencia que va de 1810 a 1819, (Herrán, 2009:210) da a conocer que, en Colombia, desde la época de la Independencia, los indígenas se ubicaron en resguardos donde desarrollaron su modus vivendi, siendo posteriormente despojados de forma gradual de la tierra madre. Se ha dado a conocer que Manuel Quintín Lame, líder indígena Colombiano, a comienzos del siglo XX, se encargó de defender los derechos de los indígenas, sus tierras, apoyando la lucha contra los impuestos; connotándole como figura principal para la promoción de los derechos de los indígenas frente a la Nación.

Toda esta labor ha generado una fuerza vital a las minorías étnicas de la actualidad, permitiéndoles participar en la política a través de la asignación de curules en la Cámara de Representantes. Sin embargo, el logro de tales beneficios no resulta suficiente, cuando en su aplicación no asegura su respeto, operatividad y cumplimiento, debido a que "los gobiernos de turno han tenido que privilegiar la atención y financiamiento del conflicto armado interno” (Herrán, 2009: 210).

Los grupos como las comunidades afrocolombianas, raizales, palenqueras, del pueblo Rom y las indígenas que se encuentran en situaciones de inequidad, requieren de protección por parte del Estado, que se debe encargar de idear planes, programas y proyectos de políticas públicas que permitan la inclusión y la participación activa de estas minorías étnicas; por lo tanto es necesario,

"generar un equilibrio de las cargas para aquellas comunidades que se encuentran en situaciones de vulnerabilidad étnica y que constituyen una minoría, por lo cual el Estado tiene el poder-deber de proveer los mecanismos, procedimientos y alternativas en términos de políticas públicas que logren derivar la prevalencia de una inclusión real y material (...) para auspiciar un escenario igualitario en términos de oportunidades y beneficios" (Gallo, 2016: 51).

A propósito de lo descrito y, en el marco de los Derechos Humanos, la Constitución Política de Colombia, desde el Preámbulo expresa que el Estado Colombiano garantizará a sus integrantes el derecho a la vida, también la convivencia, el trabajo, la justicia, la igualdad, el conocimiento, la libertad y la paz. Los artículos 1, 2, 5 y 13 hacen referencia a que prima la dignidad humana, entre otros aspectos y reconoce sin discriminación alguna, la primacía de los derechos inalienables de la persona, amparando a la familia como institución básica de la sociedad. Observando el cúmulo de derechos, la Constitución se considera garantista, incluyente y defensora de los Derechos Humanos. En consecuencia, se cumple este cometido cuando el Estado promueve las condiciones para que ese derecho a la igualdad sea real y efectivo, adoptando medidas en favor de grupos discriminados o marginados. Cabe recordar los aportes de Habermas (1999), cuando dice que "el multiculturalismo implica la coexistencia de formas de vida en igualdad de derechos” (Figuera \& Sarmiento 2016: 211). 
En su artículo 7, la Constitución Política de Colombia de 1991 reconoce que el Estado debe proteger la diversidad étnica y cultural de la nación colombiana. Semper (2006: 762) explica que la carta magna no solo se limitó a consagrar los derechos materiales de la comunidad indígena, sino que los desarrolló en materia de dignidad humana, libertad e igualdad, creando algunos instrumentos jurídicos procesales que permitieran su concreción como la acción de tutela, confiriendo la guarda de la integridad y supremacía de la Constitución a la Corte Constitucional. A su vez, los artículos 63, 329 y 330 constitucionales, esclarecen que se autorizó la conformación de entidades territoriales, estableciendo que los resguardos son de propiedad colectiva, inalienable, imprescriptible e inembargable.

En el art. 246 de la Constitución Política de Colombia de 1991 se reconoce que los pueblos indígenas podrán ejercer sus funciones jurisdiccionales en su territorio, de conformidad con sus propias normas y procedimientos con la salvedad de que no sean contrarios a la Constitución y Leyes de la República, gozando así de autonomía y reconociéndoseles el derecho de autodeterminación o de libre determinación de los pueblos, el derecho que se le otorga a los indígenas de decidir su propia forma de gobierno, sin intervenciones externas buscando así su propio desarrollo a nivel económico, social y preservando su identidad cultural.

A partir de Semper (2018a:98), se hace un reconocimiento a las normas internacionales que protegen a los indígenas y connota la importancia del Bloque de Constitucionalidad artículo 93 de la Constitución Política de Colombia de 1991, por medio del cual los tratados internacionales suscritos por Colombia prevalecen en el orden interno, es decir que son vinculantes, destacando la Corte Interamericana de Derechos Humanos, la cual es de suma importancia para los indígenas, en cuanto a la defensa de sus derechos.

De esta forma, la protección internacional de derechos humanos igualmente está respaldada por la Organización Internacional del Trabajo (OIT), la cual ha acordado múltiples convenios como el 107(1957), que se propuso integrar a los aborígenes y garantizarles su derecho a la protección e integración, además del Convenio 169 que en su artículo 14 protege los derechos de la tierra ocupada por los pueblos indígenas, a fin de preservar la propiedad como una función social.

De igual manera, en ánimo de contribuir a la preservación de los derechos humanos de las comunidades referenciadas, también existen otros organismos y convenios, entre los cuales se destacan, la Declaración de las Naciones Unidas sobre los derechos de los Pueblos Indígenas -UNDRIP-, el Pacto Internacional sobre los Derechos, Civiles y Políticos - PIDCP-, la Convención para la Eliminación de todas las Formas de Discriminación Racial - CEFDR - y la Convención Americana sobre los Derechos Humanos - CADH. Es de destacar que "cada vez que se explotan los recursos minerales en el territorio de los indígenas y se sobrepasan las necesidades propias, se suelen poner en riesgo los derechos fundamentales a la vida y la integridad étnica, social, cultural y económica" 
Haciendo una revisión de la jurisprudencia en materia de Desplazamiento Forzoso mediante Auto 091 de 2017, la Corte Constitucional protegió los derechos a la vida, integridad personal y autonomía de los integrantes de los pueblos indígenas y afrocolombianos desplazados o en riesgo de desplazamiento en los autos A.004/09 y A.005/09, por lo que procede a hacer seguimiento a las acciones adelantadas por parte del Gobierno Nacional, para la superación del estado de cosas inconstitucional declarado mediante sentencia T-025 de 2004, que verifica las medidas adoptadas para atender y proteger a las comunidades étnicas de la región del Bajo San Juan y Bajo Calima (Valle del Cauca y Chocó), en el marco del seguimiento en relación con los desplazamientos forzosos. Por designación del Estado, en visita realizada entre el 20 y el 24 de septiembre de 2016, se verificó que aún persiste el conflicto al interior de los territorios étnicos, emergencias humanitarias sobre las comunidades indígenas y afrodescendientes que habitan esta zona, la presencia, control $\mathrm{y}$ disputa territorial entre distintos actores armados ilegales.

Se ha podido constatar que la Nación busca por diferentes vías preservar el derecho constitucional a la vida y las condiciones de existencia de las minorías étnicas. Es por ello que hace un seguimiento especial al cumplimiento de las medidas adoptadas para atender y proteger estas comunidades. Se puede inferir, que a toda la problemática subyacente en los desplazamientos forzosos de los indígenas, se suma su coexistencia con las empresas instaladas en su hábitat para la explotación de los recursos naturales no renovables, exacerbando las condiciones para la continuidad de una vida digna, que no siempre logra ser copartícipe de la retribución legal que tales empresas hacen al Estado.

De los aportes de Lemos y Pedraza (2015:4), se pudo conocer que el desarrollo de la fracturación hidráulica o hidrofracturación (fracking), es un método de extracción de hidrocarburos que consiste en la fracturación de la roca para permitir la salida de los mismos, acceder a nuevos yacimientos y extraer mayor cantidad. La finalidad del Estado al permitir el uso de esta técnica, es fomentar mayores ingresos económicos, generar altos rendimientos indispensables para el desarrollo del país, aumentar puestos de trabajo, reducción de costos, disminución de la inequidad socio económicas de la población, y no depender de otros países. No obstante, la técnica en sí misma causa importantes daños al medio ambiente, contaminando las aguas y dejando una condición que pudiera propiciar movimientos de tierra como los deslaves, entre otros.

Esta técnica de explotación industrial, al producir un deterioro sistemático de todo el medio ambiente, deja prácticamente desprovisto de condiciones habitables a buena parte de las comunidades indígenas que cohabitan en tales territorios, propiciando un desplazamiento forzoso, cuando sus habitantes van en busca de otros lugares que puedan proveerles los recursos naturales para una vida digna. 
La Corte Constitucional, mediante Sentencia C-123 de 2014, hace relación a la protección al medio ambiente en Colombia, preceptuando que este se debe reconocer como principio y como derecho del cual son titular todas las personas, quienes a su vez al momento de verse perjudicadas están legitimadas para disponer de los medios legales para su protección y conservación. Al Estado se le imponen unos deberes correlativos entre los que se destacan la protección de la diversidad e integridad del medio ambiente, salvaguardar las riquezas naturales de la Nación, conservar las áreas de especial importancia ecológica, fomentar la educación ambiental, planificar el manejo y aprovechamiento de los recursos naturales para garantizar su conservación, restauración o sustitución, prevenir y controlar los factores de deterioro ambiental, imponer las sanciones legales y exigir la reparación de los daños causados al ambiente así como cooperar con otras naciones en la protección de los ecosistemas situados en las zonas de frontera. Plantea la tesis de que las actividades de tipo económica que puedan tener consecuencias en el medio ambiente, deben realizarse teniendo en cuenta los principios de conservación, sustitución y restauración del ambiente.

El presidente de la máxima industria petrolera de la nación, anunció a los medios de comunicación que para el 2019 se pondría en marcha un plan piloto de Fracking en la zona del Magdalena Medio Santandereano, en donde se estima que hay entre 2 mil y 7 mil millones de barriles de reserva. Se ha dejado conocer que los equipos de alta tecnología previstos se basan en el fracturamiento hidráulico en yacimiento no convencionales que contribuirían al desarrollo de la economía en Colombia. Blanco, S (2018 RCN Radio).

No obstante, el Consejo de Estado de Colombia mediante auto del 8 de noviembre de 2018, suspendió los actos administrativos por medio de los cuales el Gobierno Nacional fijó los criterios para la exploración y explotación de hidrocarburos en yacimientos no convencionales a través de la técnica mencionada. Se pudo concluir que la autorización en Colombia para la aplicación del fracking puede acarrear una afectación grave al medio ambiente y a la salud humana, razón por la que muchos países han ordenado su prohibición. Por tal motivo, se consideró hacer uso de la medida cautelar de suspensión provisional, para que antes de que se aplique esta técnica, se investigue de una manera más amplia cuáles serían los daños potenciales y riesgos, cómo sería el manejo adecuado de éstos, sus posibles efectos y medidas a tener en cuenta.

Otro de los aspectos considerados en la Constitución Política de Colombia, inherentes a la discriminación positiva, reside en los artículos 356 y 357, en donde la ley establece un tratamiento especial para los indígenas, considerando al Sistema General de Participaciones - SGP. Este sistema establece que los recursos que la Nación transfiere por mandato Constitucional a las entidades territoriales y a los resguardos indígenas, para la financiación de algunas de sus necesidades, está compuesto por cuatro asignaciones como son: educación, salud, agua potable y saneamiento básico. 
Así mismo, la ley 1530 de mayo 17 de 2012, en sus artículos 40 y 41 que regula la organización y el funcionamiento del SGR, destina de los recursos de asignaciones directas superiores a 2.000 SMLMV, al menos el 3\% de estos recursos para proyectos de inversión con enfoque diferencial en esa población indígena, y un 1\% para proyectos de inversión. Aun así se puede observar que el porcentaje asignado de forma exclusiva a estas minorías poblacionales, sigue siendo insuficiente con respecto a sus necesidades, derivadas en gran medida del deterioro ocasionado a su hábitat.

\section{Marco normativo del Sistema General de Regalías (SGR)}

A través del Sistema General de Regalías (SGR) en Colombia y con los aportes de Benítez, (2013a: 153), se deja conocer que, aunque en la constitución de 1886 no se menciona el término regalías sin embargo el art. 202 numerales 2 y 3 hacía referencia a la propiedad que tenía la República de Colombia sobre los baldíos, minas y salinas y que constituían dominio de la Nación de igual modo respecto de minas oro, plata, platino y piedras preciosas que existían en el territorio. (Consejo Nacional Constituyente de Colombia, 1986).

Abordando parte de la historia contemporánea de la industria petrolera en Colombia, explica que en el año 1905 el Estado colombiano otorga las dos primeras concesiones petroleras denominadas La Concesión de Mares, con una duración de treinta años. Con ello, se crea la empresa Ecopetrol en 1951 "como una empresa industrial y comercial del Estado, que sería la empresa que administraría los bienes que iban a revertir a la Nación" (Gutiérrez, 2011:1). Posteriormente nace la ley 20 de 1968 y en su artículo 12, autoriza al gobierno para declarar reserva nacional cualquier área petrolífera del País y entregarla en concesión a Ecopetrol, con el fin de que esta empresa realizara la explotación de los recursos con capital bien nacional o extranjero.

En la nueva Constitución Política de Colombia en 1991 aparece por primera vez el concepto de regalías y es así como fue ratificada la propiedad del Estado sobre los recursos naturales no renovables, reuniéndose dos iniciativas: a) La propiedad del estado como un todo donde los departamentos y municipios productores tienen participación. b.) El otorgamiento del beneficio particular a los entes territoriales productores, para no desconocer el impacto que la explotación de estos recursos tiene sobre el territorio. (Candelo, Duran, Espitia, Garces \& Restrepo: 2010: 149).

Se reconoce entonces que el Sistema General de Regalías en Colombia (SGR), tiene origen Constitucional en los artículos 360 y 361 (Constitución Política de Colombia, 1991), siendo modificado por el acto legislativo 05 de 2011 y que a su vez fue regulado por la ley 1530 de 2012 , por medio de la cual se regula la organización y el funcionamiento del SGR, que define las regalías como la contraprestación que recibe el Estado por la explotación de los recursos naturales no renovables, por los cuales se entiende todos aquellos bienes que nos ofrece la naturaleza a los seres humanos sin la intervención del hombre, entre esos tenemos los metales preciosos, oro, plata y platino e hidrocarburos, entre otros, cuya producción se extingue por el transcurso del tiempo. (Becerra, 2015: 
2). Este conjunto de ingresos, asignaciones, órganos, procedimientos y regulaciones constituye el Sistema General de Regalías (Artículo 360. Constitución Política de Colombia, 1991).

Esta reforma representó una nueva distribución de los recursos que provenían del SGR, debido a que anteriormente hubo dos tipos de asignaciones; la primera era directa, la cual consistió en otorgar el 80\% a los departamentos productores Arauca, Casanare, Cesar, la Guajira, Meta y Santander; y la segunda indirecta, la cual correspondía a un $20 \%$ que era canalizado a través del Fondo Nacional de Regalías y financiaban proyectos regionales de inversión prioritarios en todo el país, según los respectivos planes de desarrollo (Benítez, 2013b: 7). En el mismo sentido, tal como lo expresa Sánchez (2015:164), el 80\% de las regalías terminaban beneficiando solo el 17\% de la población colombiana para los años 1995 a 2010, lo cual generó un aumento de la pobreza y la brecha entre los territorios, determinando una problemática que ha persistido vulnerando los derechos humanos de la población étnica, entre otros.

Colombia, por precepto Constitucional, establece que el Estado es propietario del subsuelo y de los recursos naturales no renovables, artículo 322. Frente a este mandato la Corte Constitucional, se pronunció en Sentencia C 010 de 2013 en el sentido de que, las regalías son propiedad del Estado, teniendo las entidades territoriales titulares derecho de participación, entendiendo por entidades territoriales conforme al art. 286 de la Constitución Política de 1991, "son los Departamentos, los Distritos, los Municipios y los Territorios Indígenas" y que contarían con autonomía administrativa. Un particular no podría realizar la explotación de los recursos hidrocarburíferos por su propia cuenta, el Estado se encuentra representado en la Agencia Nacional de Hidrocarburos -ANH, que será la encargada de la administración integral de las reservas de hidrocarburos propiedad de la Nación. Como parte de sus funciones, la ANH es a su vez es responsable de recaudar las regalías y girarlas a los beneficiarios (Decreto 1760 de 2003).

Cabe destacar los aportes de Blanco (20:83), cuando expone que la Constitución Política de Colombia permitió un avance trascendental al dar un mejor reconocimiento a las minorías étnicas, teniendo en cuenta la diversidad étnica y cultural a fin de constituir una sociedad plural y diversa. Desde el preámbulo de la Constitución Política de Colombia de 1991 se dice que todos los colombianos somos iguales ante la ley, Colombia es un estado social de derecho que se funda en el respeto a la dignidad humana, en el trabajo y la solidaridad de las personas que la integran y en la prevalencia del interés general.

Ante este discurso, surge la disyuntiva de si verdaderamente tales minorías étnicas gozan adecuadamente de los derechos constitucionales antes mencionados. En este sentido, habría que revisar también si la actual aplicabilidad del SGR contribuye suficientemente a la materialización de esos derechos. Desde los aportes de Fuentes, (2018: 1), el Gobierno Colombiano por intermedio del Acto Legislativo Nro.005 de 2011, realiza una reforma en la Constitución de 1991 a los artículos 360 y 
361, relacionados con el SGR, estableciendo tres aspectos básicos: 1.- La forma en que se distribuirán los recursos. 2.- El acceso a los mismos por parte de los beneficiarios y 3.- Los criterios para el uso de las regalías.

Explican Botero, Hofman \& Hernández, (2013:30) que con la Reforma Constitucional de 2011 se formuló el SGR, basado en cuatro objetivos principales: 1) ahorro para el futuro, 2) equidad social y regional, 3) competitividad y autonomía regional, y 4) manejo probo de los recursos de regalías.

"Este nuevo sistema tiene como objetivo impulsar el crecimiento regional, aumentar la equidad entre regiones, que generen ingresos para las épocas de escasez disminuir los índices de pobreza y aumentar la competitividad, con base en criterios de equidad regional, social e intergeneracional." (Bonet \& Urrego, 2014a: 6).

\section{Organismos Colegiados de Administración y Decisión (OCAD). OCAD PAZ}

De los aportes de Bonet \& Urrego (2014b:4), se destaca que para poder acceder a los dineros productos de la explotación de los recursos naturales no renovables, se debe presentar un proyecto de inversión que permita mejorar las condiciones de vida de los departamentos, municipios y/o resguardos indígenas, raizales o comunidad Rom y que estos vayan acorde con los planes de desarrollo territoriales, los cuales se evaluarán teniendo en cuenta los criterios de viabilidad, priorización y de lo cual se encargaran los Organismos Colegiados de Administración y Decisión (OCAD).

Con el objeto de destinar estos recursos para financiar proyectos de inversión que contribuyan a los OCAD en la implementación del Acuerdo Final para la Terminación del Conflicto y la consecución de una Paz estable y duradera, se crea el Órgano Colegiado de Administración y Decisión para la PAZ (OCAD-PAZ, 2017). Este órgano se encargará de viabilizar, priorizar y aprobar los proyectos de inversión, en donde se considera el mejoramiento de la calidad de vida de los grupos étnicos, entre otros, contribuyendo de esta manera a la equidad en la distribución de los recursos hacia las comunidades más necesitadas. Estos recursos pueden contribuir a la construcción de vías terciarias, agricultura, ambiente, desarrollo sostenible, inclusión social, procesos de reconciliación, justicia, derecho y reparación a las víctimas.

El inciso quinto del parágrafo 7 transitorio del Artículo 361 de la Constitución Política señaló la conformación del OCAD PAZ. El Decreto Ley 1534 del 15 de septiembre de 2017 reglamenta el funcionamiento del OCAD PAZ y la Asignación para la Paz a que se refiere el Acto Legislativo No.4 de 2017.

Sin embargo, a pesar de los esfuerzos sostenidos por medio de los instrumentos legales antes expuestos, su aplicabilidad no ha sido suficiente para poder beneficiar dignamente a la minoría étnica 
implícita en los procesos de destino de las regalías. De lo antes expuesto, se comprende que las experiencias sumadas con el SGR han dejado entrever un panorama desfavorecedor con el destino de los dineros aportados por la actividad empresarial, en el marco de su normativa.

Se identificó que los OCAD sólo se reunían una vez al semestre, siendo insuficiente para la gestión de su proceso. A partir de ello, se encamina la optimización de los proyectos de inversión para el desarrollo de Colombia y sus regiones. En los artículos 27 y 28 del Plan Nacional de Desarrollo, se garantiza el manejo transparente, responsable y eficiente de los recursos de regalías para el fortalecimiento del desarrollo local y regional, que seguirá en cabeza de las regiones. De esta manera, el artículo 27, propone separar el concepto de viabilidad de los de priorización y aprobación. En consecuencia,

"la propuesta de la Ley del Plan es que sean los miembros del OCAD quienes voten la priorización y aprobación. Con esta reforma, según lo plantea el artículo 28, los proyectos de los OCAD Regionales, de las CAR y del OCAD Paz, serían viabilizados por los ministerios o por los departamentos administrativos cabeza de sector, lo que reducirá hasta en un $40 \%$ los tiempos de aprobación. En el caso de los OCAD departamentales y municipales, la viabilidad integral de los proyectos de inversión" (Departamento nacional de planeación DNP, 2019)

Por otra parte, cuando las empresas se consolidan en alguna región para su actividad productiva, si bien refieren un aporte para el desarrollo socioeconómico de sus habitantes, también son reconocidos los problemas de inequidad en el aspecto laboral. En relación con lo expuesto, la Asociación nacional de empresarios de Colombia ANDI (2019:4) expone que "los indígenas y afrocolombianos ocupan las posiciones más bajas en la pirámide ocupacional, es decir que están en posiciones laborales que requieren menor calificación y tienen menor remuneración que los blancos y los mestizos". Con La ley 1942 de 2018, que regula el presupuesto de ingresos del SGR, el Gobierno Nacional establece que, espera contribuir con una parte sustancial a las mejoras de la calidad de vida de las minorías étnicas, restableciendo sus derechos, de esta forma, asegurando el Estado social de derecho y el cumplimiento de los fines del mismo.

\section{CONCLUSIONES}

A fin de satisfacer las necesidades de la Población, en especial de las minorías étnicas, el Estado debe buscar algunas alternativas para disminuir las brechas sociales, buscando la igualdad de condiciones y oportunidades para los resguardos indígenas, la comunidad de raizales, afrocolombianos y gitanos o Rom, velando por sus derechos humanos y enfatizar en la necesidad de protección de los grupos étnicos por parte del Estado.

Ha sido posible constatar que a partir de la implementación del SGR, el Estado colombiano optó por conseguir una retribución adecuada de ingresos, derivados de la explotación de los recursos 
naturales no renovables y, en este sentido, se entiende que también se benefician los grupos étnicos que se encuentran en el mismo hábitat de explotación.

Comprender la vulnerabilidad de los derechos humanos de los grupos étnicos, significó reconocer que su desplazamiento forzado ha estado ligado en gran medida, por la explotación de los recursos naturales de su entorno, unido también a los problemas que conllevan los grupos delincuenciales y los alzados en armas, además del narcotráfico que transitan por la misma zona.

En el marco de los conflictos que envuelven el estado de vida y derechos de las minorías étnicas, algunas decisiones de carácter jurídico, han develado la poca consideración que se otorga a esta población, por lo cual la Corte Constitucional ha tenido que hacer un seguimiento especial para que se cumpla lo ordenado en las respectivas sentencias, con la intención de remediar la crisis de la tutela judicial efectiva ocasionada con sus desacatos, para de esta forma interrumpir la violación masiva de derechos fundamentales y descongestionar los despachos judiciales.

En medio de tales circunstancias, al inicio del SGR solo se beneficiaban los municipios y departamentos en donde se explotaban los recursos naturales no renovables y por donde se transportaban los mismos, quedando marginada gran parte de las poblaciones inmersas en el hábitat, como ha sido el caso de las minorías étnicas.

Posteriormente, las reformas del SGR se orientan hacia la equidad social y económica de todos los lugares geográficos involucrados, siendo más explícitos en relación con los grupos étnicos y la forma de retribuir las ganancias. Es por ello que se hace indispensable la vigilancia permanente de estas comunidades étnicas y la inversión de los recursos por concepto de regalías relacionados con ellas, con el objetivo de lograr un beneficio retributivo a estos territorios, teniendo en cuenta los cuatro aspectos fundamentales descritos, como lo son, educación, agua potable y saneamiento básico.

\section{REFERENCIAS BIBLIOGRÁFICAS}

Acosta, W. L. (2017). Medio ambiente y derechos humanos fundamentos teórico-jurídicos de integración en Colombia. Nova et Vétera, (26), 41-87. Recuperado de: https://dialnet.unirioja. es/servlet/articulo? codigo $=6481694$

Asociación nacional de empresarios de Colombia ANDI (2019). La inclusión de minorías étnicas: una ventaja competitiva para las empresas en Colombia. Asociación nacional de empresarios de Colombia. Bogotá: 4-5. Recuperado de http://www.andi.com.co/Uploads/Paper\%20 Minorias\%20Etnicas\%20Lectura.pdf 
Becerra-Arévalo, Y. M. (2015). Sistema general de regalías: nuevos recursos para la ciencia, tecnología e innovación en Colombia. Revista CEA, 1(1), 75-91. Recuperado de DOI: https:// doi.org/10.22430/24223182.65

Benítez Ibagué,Y. (2013). Caracterización del nuevo sistema general de regalías y su efecto fiscal en los municipios de sexta categoría del departamento de Boyacá, Colombia. Revista Finanzas y Política Económica, Vol. 5, no. 1 (ene.-jun. 2013); p. 151-178. Recuperado de: https:// hdl.handle.net/10983/18382

Blanco, J. (2005). Colombia multicultural historia del derecho a la inclusión. Diálogos De Saberes. Vol. 22(22): 81-94. Recuperado de: https://revistas.unilibre.edu.co/index.php/dialogos/article/ view/3932

Blanco, S (2018 RCN Radio). Recuperado de https://www.rcnradio.com/economia/ ecopetrol-implementara-plan-piloto-de-fracking-en-magdalena-medio

Bonet, J. y Urrego J. (2014). El Sistema General de Regalías: ¿mejoró, empeoró o quedó igual? .Documento de trabajo sobre Economía Regional, 198. Recuperado de: http://www.banrep. gov.co/sites/default/files/publicaciones/archivos/dtser_198.pdf

Botero, M., Hofman Quintero, J., \& Hernandez, D. (2016). Inequidades Territoriales en Colombia: Un Balance del Sistema General de Regalías (SGR) en el cierre de la brecha interregional. Revista Opera, $\mathrm{N}^{\circ} 17$.. 27-66. DOI: https://doi.org/10.18601/16578651.n17.03

Candelo, J., Durán Bobadilla, Y., Espitia Zamora, J., Garcés Cano, J.\& Restrepo, J. (2010). “Las regalías en Colombia y su impacto en el ámbito subnacional”. Desafíos, 22 (1): 143-203. Recuperado de: https://revistas.urosario.edu.co/index.php/desafios/article/viewFile/1292/1168

Corte Constitucional (2018). Auto 634 sentencia T 025 de 2004. [MP: Gloria Stella Ortiz Delgado]. Recuperado de: http://www.corteconstitucional.gov.co/T-025-04/AUTOS\%202018/ Auto\%20634\%20del\%2027\%20de\%20Septiembre\%20de\%202018.pdf

Departamento nacional de planeación (DANE, 2007) Colombia una nación multicultural su diversidad étnica. Recuperado de https://www.dane.gov.co/files/censo2005/etnia/sys/ colombia_nacion.pdf

Departamento nacional de planeación (DANE, 2019). Población indígena de Colombia resultados del censo nacional de población y vivienda 2018 Septiembre 16 de 2019. Recuperado en https://www.dane.gov.co/files/investigaciones/boletines/grupos-etnicos/presentacion-grupos- 
etnicos-2019.pdf

Departamento nacional de planeación DNP, 2019 En 40\% se reducirán tiempos para aprobación de proyectos con regalías. Recuperado de https:/www.dnp.gov.co/Paginas/ En-40-por-ciento-se-reducir\%C3\%A1n-tiempos-para-aprobacion-de-proyectos-con-regalias. aspx

Figuera \& Sarmiento (2016). Autodeterminación Indígena en Colombia: estudio jurídico-político del caso de la comunidad Mokana de Malambo en el Caribe Colombiano. Barranquilla, Colombia: Universidad del Norte y Grupo Editorial Ibáñez.

Fuentes Suárez, D. Y. (2018). Órganos colegiados de administración y decisión-OCAD-en el sistema general de regalías: análisis de los OCAD como modelo de toma de decisiones: disposiciones normativas vs aspectos discrecionales. Trabajo de Maestría en Gobierno y Políticas Públicas. Universidad del Externado de Colombia. Recuperado de https://bdigital. uexternado.edu.co/handle/001/1063

Gallo, E. (2016) Enfoque diferencial del Sistema General de Regalías: Una garantía para el etnodesarrollo en Colombia. Innovando en la U, Vol. 8(7): 45-55. Recuperado de: https:// revistas.unilibre.edu.co/index.php/innovando/article/view/3904/3282

Gutiérrez, J. (2011). A sus 60 años Ecopetrol está muy rejuvenecida. Revista Dinero. Recuperado de: https://www.dinero.com/negocios/articulo/60-anos-ecopetrol-esta-muy-rejuvenecida/133928

Herrán, O. (2009). Las minorías étnicas colombianas en la constitución política de 1991. Universidad Militar Nueva Granada. Prolegómenos. Derechos y Valores, Vol.12 №(24), pp 189-212. Recuperado de doi: https://doi.org/10.18359/prole.2488

Lemos González, M. (2015). La autorización del fracking en Colombia, ¿una decisión apresurada. Revista de Derecho Público, 35: 1-41. Recuperado de DOI http://dx.doi.org/10.15425/ redepub.35.2015.09

Salgado, A. (2017). Constitución y Derechos Humanos. Revista Mario Alario D'Filippo 9 (18): 21-30. Recuperado de DOI https://doi.org/10.32997/2256-2796-vol.9-num.18-2017-2051

Sánchez, P. (2015). El sistema general de regalías como instrumento de equidad territorial en Colombia. Principia Iuris. Vol. 12 (24): 159-181. Recuperado de: http://revistas.ustatunja.edu. co/index.php/piuris/article/download/1084/1050 
Semper, F (2006).Anuario de Derecho Constitucional Latinoamericano 2006. Sempre, F en Derecho indígena y Los derechos de los pueblos indígenas de Colombia en la jurisprudencia de la Corte Constitucional (761-778) Montevideo, Uruguay: Konrad Adenauer Stiftung E.V

Semper, F. (2018). Los derechos de los pueblos indígenas en Colombia. Bogotá: Editorial Temis. 